\title{
ANTIMICROBIALS Remdesivir in Children
}

\author{
K Dhanalakshmi \\ Pediatric Infectious Disease (2021): 10.5005/jp-journals-10081-1295
}

At the end of 2019, the cause for the cluster of cases of pneumonia in Wuhan, China, was identified as a new novel coronavirus-severe respiratory distress syndrome 2 (SARS-CoV-2). It subsequently spread throughout the world becoming a pandemic and it was designated as COVID-19 by the WHO. The symptoms of COVID-19 (coronavirus inclusion disease) vary from asymptomatic infection to life-threatening complications like severe pneumonia requiring mechanical ventilation, septic shock, multiorgan dysfunction, and death. ${ }^{1,2}$ While the majority of the infections in children are asymptomatic or had mild or moderate disease, the greatest risk for severe and critical disease $\mathrm{e}^{2,3}$ are elderly patients with underlying cardiovascular or respiratory problems.

\section{Therapeutic Options in COVID-19}

Since the beginning of the pandemic, there have been $>2,800$ clinical trials registered on COVID-19 interventions. Lopinavirritonavir, hydroxychloroquine, remdesivir, interferon beta 1a, and steroids are the drugs that are currently investigated by the trials. Apart from antivirals, other interventions, such as, convalescent plasma and immunomodulatory therapies, are also being evaluated. To date, no effective antiviral therapy has been approved for SARS-CoV- $2^{4}$ and supportive care is the mainstay of treatment.

Remdesivir is one of the potential therapeutic options which was evaluated extensively. The Gilead Sciences developed remdesivir (GS-5734). It emerged from collaboration between Gilead, the U.S. Centers for Disease Control and Prevention, and the U.S. Army Medical Research Institute of Infectious Diseases. Even though most of the clinical trials on remdesivir involved only adults, parenteral remdesivir has been approved through emergency use authorization and compassionate use by the US Food and Drug Administration in pediatric patients and by the European Medicines Agency for adolescent patients ( $\geq 12$ years). ${ }^{5}$ Recently, the interim results of Solidarity Trial were published. Since, remdesivir has no impact on the mortality, time for clinical improvement, need for mechanical ventilation, and other patient outcomes, a conditional recommendation was issued against the use of remdesivir by WHO in hospitalized patients. ${ }^{6}$ Even though its role is uncertain, remdesivir has been widely used as a potentially effective treatment for COVID-19.

\section{Drug Targets for Treatment}

Severe respiratory distress syndrome 2 is an enveloped RNA virus that is single-stranded. It binds to angiotensin-converting enzyme (ACE 2) receptors through structural spike (s) protein and enters the cell. Using various endosomes and host cell receptors, the virus enters the cell after the receptor binding. Cell entry via the $S$ protein is facilitated by TMPRSS2, host type 2 transmembrane serine protease. ${ }^{7}$ Viral proteins are synthesized inside the cell, which codes for the replicase-transcriptase complex. Using its RNA-dependent
Department of Pediatrics, Kanchi Kamakoti Childs Trust Hospital, Chennai, Tamil Nadu, India

Corresponding Author: K Dhanalakshmi, Department of Pediatrics, Kanchi Kamakoti Childs Trust Hospital, Chennai, Tamil Nadu, India, Phone: +91 8939345600, e-mail: drkdhana77@gmail.com

How to cite this article: Dhanalakshmi K. Remdesivir in Children. Pediatr Inf Dis 2021;3(2):74-76.

Source of support: Nil

Conflict of interest: None

RNA polymerase, the virus synthesizes RNA. Structural proteins ate then synthesized leading to the assembly and release of viral particles from the host cell. ${ }^{8,9}$ These steps involved in the life cycle of the virus provides potential targets for drug therapy.

\section{Mechanism of Action of Remdesivir}

It inhibits viral RNA polymerases by intracellularly metabolizing the analog of adenosine triphosphate. Once remdesivir has been mistakenly incorporated into the chain, the synthesis of RNA is terminated. ${ }^{10,11}$

Antiviral activity: It has been shown effective against many viruses including Filoviruses (e.g., Ebola), Nipah virus, respiratory syncytial virus, and coronaviruses (e.g., SARS-CoV-1 and MERSCoV). ${ }^{12,13}$

With a half-maximal effective concentration (EC-50) of $0.01 \mu \mathrm{M}^{14}$ the replication of the virus was inhibited effectively in vitro in the primary human airway epithelial cultures. ${ }^{14}$ This antiviral activity appears to be virus-specific. In SARS-CoV-infected mice, treatment with remdesivir significantly reduced lung viral load and loss of pulmonary function. ${ }^{14}$

It was also noted that chloroquine can antagonize remdesivir. When both were co-incubated in HEp-2 cells infected with a respiratory syncytial virus, reduced remdesivir triphosphate formation and higher remdesivir EC-50 values were observed in normal human bronchial epithelial cells with increasing doses of chloroquine. ${ }^{15}$ Hence, the co-administration of chloroquine phosphate or hydroxychloroquine sulfate and remdesivir is not recommended. ${ }^{15}$

\section{Therapeutic Efficacy}

Early in the pandemic, 237 patients (158 assigned to remdesivir and 79 to placebo) were enrolled in China by Wang et al. and showed that with the use of remdesivir, the time to improvement is shorter: 21.0 days $[95 \%$ confidence interval $(\mathrm{Cl}), 13.0-28.0]$ in the remdesivir group and 23.0 days $(95 \% \mathrm{Cl}, 15.0-28.0)$ in the placebo group. ${ }^{16}$ Owing to local control of the outbreak, the trial was not completed and statistical demonstration of the significant clinical benefits of remdesivir was not done. 
A multinational, randomized, placebo-controlled trial (the Adaptive COVID-19 Treatment Trial ACTT-1) gave the recent recommendations on the use of remdesivir. ${ }^{17}$ The trial had 1,062 hospitalized adults with the involvement of lower respiratory tract and they were assigned randomly to receive remdesivir or placebo in 1:1 ratio.

Shorter time to recovery was noted in patients in the remdesivir group than patients in the placebo group (median, 10 days, as compared with 15 days; rate ratio for recovery, $1.29 ; 95 \% \mathrm{Cl}, 1.12-1.49$; $p<0.001)$. Patients with tachypnea $/ \mathrm{SpO}_{2}<94 \%$ in room air/requiring supplemental oxygen/requiring mechanical ventilation were classified as severe disease. In this severe disease group, the recovery time was 11 days, as compared to 18 days (rate of ratio for recovery, $1.31 ; 95 \% \mathrm{Cl}, 1.12-1.52$ ). Maximum benefit (rate ratio for recovery) was noted among patients requiring only supplemental oxygen when compared with patients requiring high-flow nasal cannula (HFNC), mechanical ventilation, or extracorporeal membrane oxygenation (ECMO) (recovery rate, $1.45 ; 95 \% \mathrm{Cl}, 1.18-1.79$ ). Additional secondary endpoints include that the improvement time is shorter in one or two ordinal scale categories, time to discharge is shorter, and the initial length of hospital stay is shorter (median, 12 vs 17 days). Allcause mortality with remdesivir and placebo was 11.4 and $15.2 \%$, respectively (hazard ratio, $0.73 ; 95 \% \mathrm{Cl}, 0.52-1.03$ ).

One of the other largest clinical trials launched by WHO is the Solidarity Trial enrolling patients over 30 countries. Remdesivir, hydroxychloroquine, lopinavir, ritonavir, and interferon beta 1a were the study drugs included. Locally available study drugs were randomized equally among hospitalized patients with COVID-19. The trial concluded that none of the study drugs affected the main outcomes of mortality, initiation of ventilation, and duration of hospitalization.

Even though the ACTT-1 trial showed a moderate reduction in time to recovery, Solidarity Trial showed no benefit in the initiation of ventilation and in the time to discharge. ${ }^{18}$

\section{Dose of Remdesivir}

It is available as a solution and/or lyophilized powder and it is intravenously administered for infusion over 30-120 minutes. The recommended dosage regimen (for adults and children $>40 \mathrm{~kg}$ ) is a single $200 \mathrm{mg}$ loading dose on the first day and from day 2 onward $100 \mathrm{mg}$ is given once daily. For pediatric patients, $<40 \mathrm{~kg} 5 \mathrm{mg} / \mathrm{kg}$ is given on day 1 and $2.5 \mathrm{mg} / \mathrm{kg}$ is given daily for the remaining days. The total duration of treatment must be at least 5 days and should not exceed 10 days.

Before the initiation of treatment with remdesivir, kidney and liver functions should be determined.

\section{Duration of Therapy}

Data from phase 3 of a randomized, open-label trial that involved 397 hospitalized patients showed similar clinical efficacy for 5 or 10 days of treatment with remdesivir. ${ }^{19}$ All the patients in the trial had confirmed SARS-CoV-2 infection, with oxygen saturation of $94 \%$ or less in room air and radiologic evidence of pneumonia. By the 14 th day, $64 \%$ of patients in the 5 -day group and $54 \%$ in the 10 -day group showed a clinical improvement of 2 points or more on the ordinal scale. Patients in the 10-day group had a clinical status at day 14 which was similar to that among patients in the 5-day group $(p=0.14)$ after adjustments of baseline clinical status.

For patients who do not improve after 5 days of receiving remdesivir, the optimal duration of therapy is not clear. If there is no improvement after 5 days, extending the duration of treatment up to 10 days is considered by some experts. ${ }^{20}$

\section{Pharmacokinetics}

Remdesivir undergoes metabolic activation intracellularly to the pharmacologically active nucleoside analog triphosphate (GS443902), while the nucleoside analog GS 441524 is produced by dephosphorylation of phosphorylated metabolites. ${ }^{15,21}$

Irrespective of dose level, the peak of plasma concentrations of remdesivir was at the end of infusion and declined rapidly thereafter. The peak of plasma concentrations of the predominant circulating metabolite was 1.5-2.0 hours after the start of a 30-minute infusion. The human plasma protein binding rates of remdesivir and GS-441524 are around 88 and $2 \%$, respectively. Similarly, their median terminal half-lives were 1 and 27 hours, respectively.

Renal clearance is the main elimination pathway for GS-441524. Around 74 and $18 \%$ of the dose was recovered in urine and feces, respectively, following intravenous injection. The impact of age, sex, race, or hepatic impairment on the pharmacokinetics of remdesivir is unknown. Before starting treatment with remdesivir and during treatment, all patients should have their estimated glomerular filtrations rate (eGFR) determined as clinically appropriate, even though the pharmacokinetics of remdesivir and GS-441524 have not been evaluated in patients with renal impairment. In patients with an eGFR of $<30 \mathrm{~mL} /$ minute, it should not be given. Also, full-term neonates with serum creatinine level $1 \mathrm{mg} / \mathrm{dL}$ or greater should not be given remdesivir. ${ }^{22}$ The formulation of remdesivir contains the excipient, 3 $\mathrm{g}$ of sulfobutylether-beta-cyclodextrin sodium salt (SBECD) per 100 mg, which can trigger kidney impairment. Sulfobutylether-betacyclodextrin sodium salt can also accumulate in pediatric patients with underlying renal abnormalities [renal impairment and receiving renal replacement therapies like hemodialysis, peritoneal dialysis, and continuous renal replacement therapy (CRRT)].

It also causes an increase in aminotransferase levels; hence, before initiation of treatment and during treatment, liver function should be evaluated and monitored. ${ }^{23}$ It should not be initiated in patients with alanine aminotransferase (ALT) $\geq 5$ times, the upper limit of normal at baseline. Hence, there is an increased need for clinical trials for pediatric patients with hepatic or kidney impairment or ECMO requirements due to a lack of safety or pharmacokinetic data.

\section{Drug Interactions}

Increased intrahepatocellular concentration above the toxicity level can occur due to a combination of P-glycoprotein inhibitors (e.g., hydroxychloroquine, azithromycin, cyclosporine, tacrolimus, and amiodarone) with remdesivir. Children can have symptomatic seizures with COVID-19 infection and antiepileptic drugs have interaction with remdesivir. Strong induction of CYP3A4 and CYP2C9 by anti epileptic drugs (AEDs) (e.g., carbamazepine, phenobarbital, phenytoin, and primidone) may significantly reduce the serum concentration of remdesivir.

\section{Adverse Effects}

In clinical trials, intravenous administration of remdesivir was generally well tolerated. The common adverse events reported in adults were anemia, acute kidney injury $(\mathrm{AKI})$, pyrexia, hyperglycemia, and increased liver enzymes [ALT and/ 
or aspartate aminotransferase (AST)]. ${ }^{23}$ No deaths were directly related to remdesivir. If a patient develops signs/symptoms of liver involvement [ALT $\geq 5$ upper limit of normal (ULN), increasing conjugated bilirubin, alkaline phosphatase, or international normalized ratio (INR)] during treatment, remdesivir should be discontinued. Similarly, in patients with an eGFR of $<30 \mathrm{~mL} /$ minute, it should not be used. There is a possibility of the occurrence of hypersensitive reactions (including infusion-related reactions and anaphylactic reactions) with remdesivir, and if it occurs, slowing the infusion rates ( $\leq 120$ minutes of maximum infusion time) is considered by clinicians. Remdesivir administration should be immediately discontinued if a clinically significant hypersensitivity reaction occurs.

\section{Consideration in Children}

Most of the major trials on remdesivir have not included children and adolescents under the age of $19 .^{18}$ Therefore, the safety and effectiveness of remdesivir for the treatment of COVID-19 in pediatric patients has not been known.

However, treatment with an antiviral drug alone is not likely to be sufficient for all patients, given the high mortality associated with COVID-19. To improve the clinical outcome in patients with COVID-19, several therapeutic approaches including novel antivirals, immunomodulators, monoclonal antibodies, and combination approaches are needed.

\section{Consideration in Pregnancy and LACTATION}

In pregnant patients, the safety and effectiveness of remdesivir for COVID-19 treatment has not been evaluated. Thus, it is used only when the potential benefit justifies the potential risk to the mother and fetus. Similarly, it should be used with careful infant monitoring during breastfeeding. Infants are not likely to absorb clinically important amounts of the drug through breastfeeding because of poor oral absorption of remdesivir.

\section{References}

1. Website [Internet]. [cited 2020 Oct 22]. Available from: RodriguezMorales AJ, Cardona-Ospina JA, Gutiérrez-Ocampo E, et al. Clinical, laboratory and imaging features of COVID-19: a systematic review and meta-analysis. Travel Med Infect Dis [Internet] 2020;34:101623. Available from: http://dx.doi.org/10.1016/j.tmaid.2020.101623.

2. Website [Internet]. [cited 2020 Oct 22]. Available from: Wu C, Chen $X, C a i Y$, et al. Risk factors associated with acute respiratory distress syndrome and death in patients with coronavirus disease 2019 pneumonia in Wuhan, China. JAMA Intern Med [Internet] 2020;180(7):934-943. Available from: http://dx.doi.org/10.1001/ jamainternmed.2020.0994.

3. Website [Internet]. [cited 2020 Oct 22]. Available from: Weiss $P$, Murdoch DR. Clinical course and mortality risk of severe COVID-19. Lancet [Internet]\#\#thelancet.com\#\#2020;395(10229): 1014-1015. Available from: http://dx.doi.org/10.1016/S0140-6736(20) 30633-4.

4. Sanders JM, Monogue ML, Jodlowski TZ, et al. Pharmacologic treatments for coronavirus disease 2019 (COVID-19): a review. JAMA 2020;323(18):1824-1836. DOI: 10.1001/jama.2020.6019.

5. Frauenfelder C, Brierley J, Whittaker E, et al. Infant with SARS-CoV-2 infection causing severe lung disease treated with remdesivir. Pediatrics [Internet] 2020;146(3):e20201701. Available from: https:// pediatrics.aappublications.org/content/146/3/e20201701.abstract.
6. Dyer O. Covid-19: remdesivir has little or no impact on survival, WHO trial shows. BMJ 2020;371:m4057. DOI: 10.1136/bmj.m4057.

7. Hoffmann M, Kleine-Weber H, Schroeder S, et al. SARS-CoV-2 cell entry depends on ACE2 and TMPRSS2 and is blocked by a clinically proven protease inhibitor. Cell 2020;181(2):271-280.e8. DOI: 10.1016/ j.cell.2020.02.052.

8. Chen Y, Liu Q, Guo D. Emerging coronaviruses: genome structure, replication, and pathogenesis. J Med Virol [Internet] 2020;92(4): 418-423. Available from: http://dx.doi.org/10.1002/jmv.26234.

9. Fung TS, Liu DX. Coronavirus infection, ER stress, apoptosis and innate immunity. Front Microbiol 2014;5:296. DOI: 10.3389/ fmicb.2014.00296.

10. Website [Internet]. [cited 2020 Oct 22]. Available from: Gordon CJ, Tchesnokov EP, Feng JY, et al. The antiviral compound remdesivir potently inhibits RNA-dependent RNA polymerase from Middle East respiratory syndrome coronavirus. J Biol Chem [Internet] 2020;295(15):4773-4779. Available from: http://dx.doi.org/10.1074/ jbc.AC120.013056.

11. Website [Internet]. [cited 2020 Oct 22]. Available from: Gordon CJ, Tchesnokov EP, Woolner E, et al. Remdesivir is a direct-acting antiviral that inhibits RNA-dependent RNA polymerase from severe acute respiratory syndrome coronavirus 2 with high potency. J Biol Chem [Internet] 2020;295(20):6785-6797. Available from: http://dx.doi. org/10.1074/jbc.RA120.013679.

12. de Wit E, Feldmann F, Cronin J, et al. Prophylactic and therapeutic remdesivir (GS-5734) treatment in the rhesus macaque model of MERS-CoV infection. Proc Natl Acad Sci U S A 2020;117(12):6771-6776. DOI: 10.1073/pnas.1922083117.

13. Wang $M$, Cao R, Zhang L, et al. Remdesivir and chloroquine effectively inhibit the recently emerged novel coronavirus (2019-nCoV) in vitro. Cell Res 2020;30(3):269-271. DOI: 10.1038/s41422-020-0282-0.

14. Pruijssers AJ, George AS, Schäfer A, et al. Remdesivir potently inhibits SARS-CoV-2 in human lung cells and chimeric SARS-CoV expressing the SARS-CoV-2 RNA polymerase in mice. bioRxiv [Internet] 2020. Available from: http://dx.doi.org/10.1101/2020.04.27.064279.

15. Website [Internet]. [cited 2020 Oct 22]. Available from: Lamb YN. Remdesivir: first approval [internet]. Drugs 2020;80(13):1355-1363. Available from: http://dx.doi.org/10.1007/s40265-020-01378-w.

16. Wang $Y$, Zhang D, Du G, et al. Remdesivir in adults with severe COVID19: a randomised, double-blind, placebo-controlled, multicentre trial. Lancet 2020;395(10236):1569-1578. DOI: 10.1016/S01406736(20)31022-9.

17. Website [Internet]. [cited 2020 Oct 23]. Available from: Remdesivir for the Treatment of Covid-19 — Final Report | NEJMwww.nejm.org > doi > full > NEJMoa2007764 Oct 8.

18. 2020 Consortium WST, WHO Solidarity Trial Consortium Pan H, Peto $\mathrm{R}$, Karim QA, et al. Repurposed antiviral drugs for COVID-19-interim WHO SOLIDARITY trial results [internet]. MedRxiv 2020. Available from: http://dx.doi.org/10.1101/2020.10.15.20209817.

19. Website [Internet]. [cited 2020 Oct 23]. Available from: Goldman JD, Lye DCB, Hui DS, et al. Remdesivir for 5 or 10 days in patients with severe Covid-19 [internet]. N Eng J Med 2020;383(19):1827-1837. Available from: http://dx.doi.org/10.1056/nejmoa2015301.

20. Fda US, Fact sheet for health care providers: emergency use authorization (EUA) of remdesivir (GS-5734 $4^{\mathrm{TM}}$ ). 2020.

21. Website [Internet]. [cited 2020 Oct 23]. Available from: Humeniuk R, Mathias A, Cao H, et al. Safety, tolerability, and pharmacokinetics of remdesivir, an antiviral for treatment of COVID-19, in healthy subjects. Clin Transl Sci [Internet] 2020. Available from: https://onlinelibrary. wiley.com/doi/abs/10.1111/cts.12840.

22. Yalçın N, Demirkan K. COVID-19 and remdesivir in pediatric patients: the invisible part of the iceberg. Pediatr Res [Internet] 2020. Available from: http://dx.doi.org/10.1038/s41390-020-01109-7.

23. Website [Internet]. [cited 2020 Oct 23]. Available from: Website [Internet]. [cited 2020 Oct 23]. Available from: Beigel JH, Tomashek KM, Dodd LE. Remdesivir for the treatment of Covid-19 - preliminary report. Reply N Engl J Med [Internet] 2020;383(10):994. Available from: http://dx.doi.org/10.1056/NEJMc2022236. 